\title{
Compartment syndrome as a result of incorrect treatment
}

\author{
Seyyed Mahdi Mirhamidi', Davood Soroosh ${ }^{2}$, Alireza Abadi ${ }^{3}$, Elahe Fahimi ${ }^{3}$ \\ 'Department of Orthopedic, Sabzevar University of Medical Sciences, Sabzevar, Iran \\ 2Department of Forensic Medicine, Sabzevar University of Medical Sciences, Sabzevar, Iran \\ ${ }^{3}$ Department of Emergency Medicine, Sabzevar University of Medical Sciences, Sabzevar, Iran
}

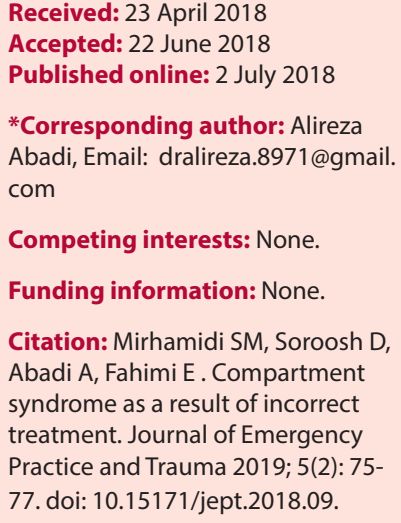

Competing interests: None.

Funding information: None.

Citation: Mirhamidi SM, Soroosh D, Abadi A, Fahimi E. Compartment syndrome as a result of incorrect treatment. Journal of Emergency Practice and Trauma 2019; 5(2): 75-

77. doi: $10.15171 /$ jept.2018.09.

\begin{abstract}
Compartment syndrome is a rare vascular disorder and an orthopedic emergency caused by high intramuscular pressure following bone fractures and some other etiologies. It mostly involves extremities, but can affect other parts of the body. The syndrome is diagnosed based on extensively varying signs and symptoms including feeling pain, pallor, pulselessness, and some other signs among patients. It causes irreversible complications that may even lead to death in some cases. If not treated promptly, muscle and nerve necrosis, myoglobinuria, and ultimately extremity gangrene and renal failure may occur. Timely diagnosis requires close attention to patient's signs and symptoms and repeated measurements of pressure in the compartment. The reported case was an 11-yearold girl suffering from a closed double fracture in her forearm following blunt trauma. Inappropriate treatment done by a local therapist resulted in compartment syndrome. The aim of this study was to obtain an accurate knowledge on it and to do timely action when facing with this syndrome.
\end{abstract}

Keywords: Compartment syndrome, Volkmann's syndrome, Treatment, Complications

\section{Introduction}

Acute compartment syndrome (ACS) is an orthopedic emergency characterized by an increase in intracompartmental pressure and disturbance in the perfusion of the compartment (1). This is a rare complication related to vascular disorder of Ehlers-Danlos syndrome (EDS) (2). The complications include neuromuscular damage, Volkmann's ischemia, rhabdomyolysis, acute renal injury, infection, and even death (3). Volkmann's syndrome was reported by Volkmann in 1869 (4). The muscles are surrounded by a hard fascicular sheath forming compartment space. Involved in trauma and crush injury, muscles are swollen, and the intra-compartmental pressure increases. It, in turn, causes muscle, vessels, and nerves to be injured in the compartment (5). This complication is observed most commonly in the forearm and tibialis anterior, with a wide variety of clinical symptoms (6). The main etiology of this lesion is unknown, but contraction of the vessels and stopping the blood flow are generally due to the increased intra-compartmental pressure following trauma and bone fractures and inappropriate casting. The most frequent cause in Iran is tight forearm bandage or casting by unskilled people (4).

\section{Case Presentation}

The patient was an 11-year-old girl who had referred to a local therapist due to a double non-displaced fracture in the distal radius and ulna of her left arm four days before referring to the orthopedic clinic when she fell from height (Figure 1). The patient was put under traditional unscientific treatments including improper reduction and bandaging. Her upper extremity began to swell from the elbow to the fingers on the next day. Subsequently, numerous juicy blisters without itching appeared in the dorsal forearm, hand, and fingers. The patient did not give a history of insect bites and drug allergy, and took no medication except tetracycline and zinc oxide ointments. The patient referred to the orthopedic clinic on the fourth day of the fracture. In the examination, the very large blisters containing clear, non-purulent fluid in the left dorsal forearm and hand with positive trans-illumination test were observed (Figure 2). The sensation and movement of the injured limb revealed that the active movements of the fingers had become very limited, and the patient was unable to do her wrist extension. Furthermore, the passive movements of her wrist and fingers were painful. In the neural examination, the radial, median, and ulnar peripheral nervous sensation were found to be impaired; 


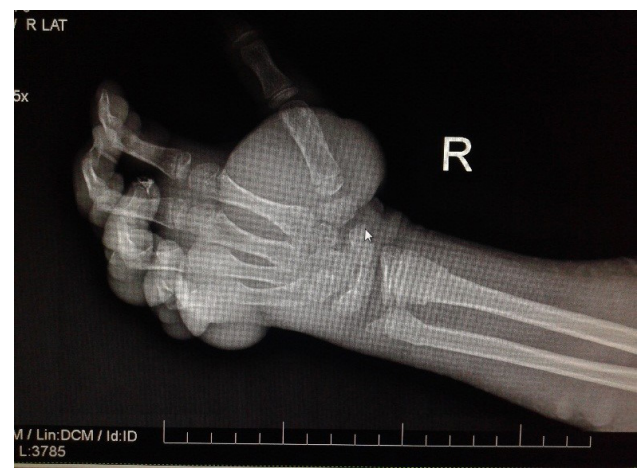

Figure 1. A double non-displaced fracture in the distal radius and ulna of her left arm.

however, the pulse of the radial artery was felt, the limb perfusion was established, and the capillary filling of the fingers was evaluated to be less than two seconds.

\section{Discussion}

ACS is an orthopedic emergency that is often seen in the extremities, but it can occur in other parts of body. As observed in $75 \%$ of cases, bone fracture is the most common etiology of the syndrome (7). CiemniewskaGorzela et al in their study reported that a 55-year-old woman was admitted to a hospital for her right hip joint injury. She then underwent arthroscopy and necessary medical treatment. After the operation, the patient suffered from abdominal pain and tenderness. The intraabdominal free fluid was seen by abdominal ultrasound and other diagnostic methods, and finally she was put under treatment of abdominal compartment syndrome (8). Other causes of this syndrome include coagulation disorders, anticoagulant therapy, and even biting of animals (Table 1) (9). The most common etiologies of compartment syndrome in the upper extremity of adult and pediatric patients are distal radius and humerus supracondylar fractures, respectively (1); however, the reported case in our study was an 11-year-old girl with the compartment syndrome due to the distal radius and ulna fracture. This syndrome is diagnosed based on the

Table 1. Causes of Acute Compartment Syndrome

\begin{tabular}{|c|c|}
\hline Internal Causes & External Causes \\
\hline Hematoma/coagulopathy & Circumferential cast or dressing \\
\hline Fracture & Burn eschar \\
\hline Overexertion of muscles (seizure) & $\begin{array}{l}\text { Military anti-shock trousers } \\
\text { (MAST) }\end{array}$ \\
\hline Post-ischemic time/reperfusion & $\begin{array}{l}\text { Compression from prolonged } \\
\text { immobility }\end{array}$ \\
\hline \multicolumn{2}{|l|}{ Animal bite/envenomation } \\
\hline \multicolumn{2}{|l|}{ Soft tissue injury } \\
\hline \multicolumn{2}{|l|}{ Vascular injury } \\
\hline \multicolumn{2}{|l|}{ Deep venous thrombosis } \\
\hline Idiopathic & \\
\hline
\end{tabular}

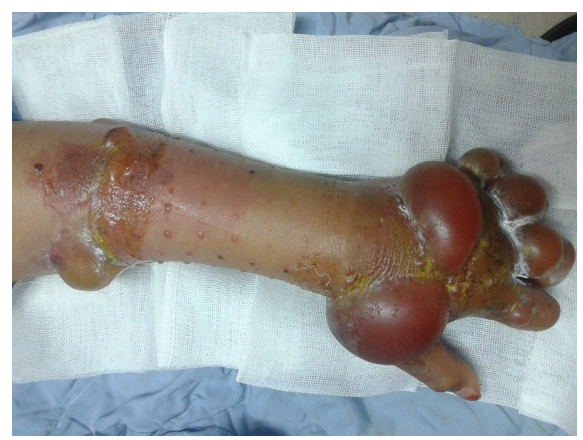

Figure 2. The very large blisters in the left dorsal forearm and hand.

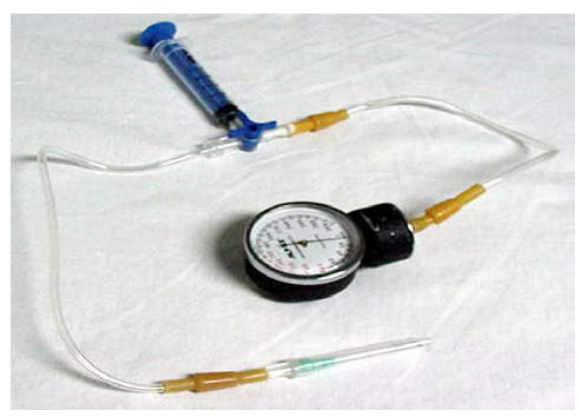

Figure 3. Stryker needle with a manometer for measuring intracompartmental pressure

clinical suspicion and the patient's symptoms (2) including pain, pallor, paresis, paresthesia, and pulselessness, represented as $5 \mathrm{P}$. Our patient had all these symptoms except pulselessness (the distal radial pulse was felt). The distal pulse does not essentially confirm lack of the compartment syndrome, since neuromuscular injuries may progress in the presence of distal limb pulses, and lack of pulse indicates end-stage and severe muscular necrosis (10). The progression of this syndrome takes place in three stages: I. Pain, swelling, and paresthesia of the affected organ; II. The neurological deficits, sensory and motor disorders, and local muscle necrosis with loss of distal limb pulse; and III. A severe necrosis in muscle and skin (5). According to the literature, normal intra-compartmental pressure ranges from 0 to $5 \mathrm{~mm}$ $\mathrm{Hg}$. When the pressure exceeds $10 \mathrm{~mm} \mathrm{Hg}$, the venous circulatory system becomes impaired, and the pressure above $20 \mathrm{~mm} \mathrm{Hg}$ leads to the ischemia. The pressure higher than $30 \mathrm{~mm} \mathrm{Hg}$ lasting more than eight hours results in irreversible neuromuscular damages $(5,6)$. The intra-compartmental pressure can be measured by means of Stryker needle or a manometer in unconscious patients with traumatic head injury or multiple traumas (Figure 3). The intra-compartmental pressure needs to be measured in severe cases (1). Also, in these cases, rhabdomyolysis, myoglobinuria, and hyperkalemia should not be ignored in para-clinical examination, because this syndrome is idiopathic, and the patients have no symptoms in 
some cases. Therefore, the syndrome diagnosis can be done by examining the para-clinical manifestations in asymptomatic cases.

Based on the literature, the selective treatment of the syndrome is fasciotomy with a specific indication, in such a way that fasciotomy can be applied in the symptomatic cases with intra-compartmental pressure higher than $30 \mathrm{~mm} \mathrm{Hg}$, or in the asymptomatic cases with intracompartmental pressure higher than $40-50 \mathrm{~mm} \mathrm{Hg}$. The limb, in mild cases, can be immobilized and placed at the previous level. Liquids may be prescribed, and the patient's urine may be basified in order to prevent acute renal complications (6). The fasciotomy is performed in several methods, which were described in detail in Gousheh's study (4). Since our patient was referred four days after fracture with resulting complications, surgery was not required according to a specialist diagnosis. Rather, physiotherapy was conducted to restore the limb's function and reduce the related complications.

The findings of this study demonstrated that timely diagnosis of ACS requires the physicians to pay close attention to the symptoms of the patients. The syndrome is diagnosed based on the clinical suspicion and careful examination, and if necessary, other diagnostic procedures. In dealing with these kinds of cases, the patient should be referred to an equipped healthcare center or specialized orthopedic clinic as soon as possible. Moreover, the public should be aware of the complications of the syndrome in order that they will not refer to local therapists who are unable to treat it appropriately.

\section{Authors' contributions}

Case treatment and identification: SMM. Writing, editing, and submitting: DS. Data and information completing: $\mathrm{AA}$ and $\mathrm{EF}$.

\section{Ethical issues}

A written consent form was obtained from the patient.

\section{References}

1. Smith K, Wolford RW. Acute idiopathic compartment syndrome of the forearm in an adolescent. West J Emerg Med 2015; 16(1): 158-60. doi: 10.5811/westjem.2014.9.23019.

2. Barajas BD, Sun A, Rimoin DL, Reinstein E. Recurrent compartment syndrome in a patient with clinical features of a connective tissue disorder. Am J Med Genet A 2013; 161A(6): 1442-6. doi: 10.1002/ajmg.a.35894.

3. Newman PA, Deo S. Non-traumatic compartment syndrome secondary to deep vein thrombosis and anticoagulation. BMJ Case Rep 2014; 2014. doi: 10.1136/ bcr-2013-201689.

4. Gousheh J. The treatment of war injuries of the brachial plexus. J Hand Surg Am 1995; 20(3 Pt 2): S68-76.

5. Tiwari A, Haq AI, Myint F, Hamilton G. Acute compartment syndromes. Br J Surg 2002; 89(4): 397-412. doi: 10.1046/j.0007-1323.2002.02063.x.

6. Beigi A, Taheri H, Sehhat S. Acute Compartment Syndrome. Iranian Journal of Surgery 2006; 14(2):1-7. [In Persian].

7. Perron AD, Brady WJ, Keats TE. Orthopedic pitfalls in the ED: acute compartment syndrome. Am J Emerg Med 2001; 19(5): 413-6. doi: 10.1053/ajem.2001.24464.

8. Ciemniewska-Gorzela K, Piontek T, Szulc A. Abdominal compartment syndrome--the prevention and treatment of possible lethal complications following hip arthroscopy: a case report. J Med Case Rep 2014; 8: 368. doi: 10.1186/17521947-8-368.

9. McQueen MM, Gaston P, Court-Brown CM. Acute compartment syndrome. Who is at risk? J Bone Joint Surg Br 2000; 82(2): 200-3.

10. Ulmer T. The clinical diagnosis of compartment syndrome of the lower leg: are clinical findings predictive of the disorder? J Orthop Trauma 2002; 16(8): 572-7. 